\title{
MICADO: the E-ELT Adaptive Optics Imaging Camera
}

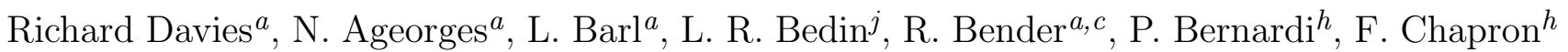
Y. Clenet $^{h}$, A. Deep ${ }^{d}$, E. Deul ${ }^{d}$, M. Drost ${ }^{f}$, F. Eisenhauer ${ }^{a}$, R. Falomo ${ }^{g}$, G. Fiorentino ${ }^{e}$, N. M. Förster Schreiber ${ }^{a}$, E. Gendron ${ }^{h}$, R. Genzel ${ }^{a}$, D. Gratadour ${ }^{h}$, L. Greggio $^{g}$, F. Grupp ${ }^{c}$, E. Held ${ }^{g}$, T. Herbst ${ }^{b}$, H.-J. Hess ${ }^{c}$, Z. Hubert ${ }^{h}$, K. Jahnke $^{b}$, K. Kuijken ${ }^{d}$, D. Lutz ${ }^{a}$, D. Magrin ${ }^{g}$,

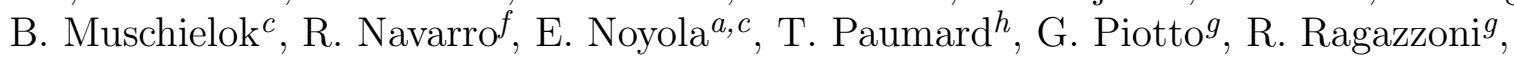

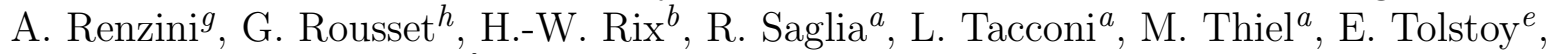
S. Trippe ${ }^{i}$, N. Tromp ${ }^{f}$, E. A. Valentijn ${ }^{e}$, G. Verdoes Kleijn ${ }^{e}$, and M. Wegner ${ }^{c}$

${ }^{a}$ Max Planck Institute for extraterrestrial Physics, Postfach 1312, 85741 Garching, Germany

${ }^{b}$ Max Planck Institute for Astronomy, Königstuhl 17, 69117 Heidelberg, Germany

${ }^{c}$ Munich University Observatory, Scheinerstrasse 1, 81679 München, Germany

${ }^{d}$ Leiden Observatory, Leiden University, 2300RA Leiden, Netherlands

${ }^{e}$ Kapteyn Astronomical Institute, University of Groningen, 9700AV Groningen, Netherlands

${ }^{f}$ NOVA-ASTRON, P.O.Box 2, Dwingeloo, Netherlands

${ }^{g}$ INAF - Astronomical Observatory of Padova, Vicolo dell'Osservatorio 5, 35122 Padova, Italy

${ }^{h}$ LESIA, Observatoire de Paris, 5 place Jules Janssen, 91195 Meudon cedex, France

${ }^{i}$ IRAM, 300 rue de la Piscine, 38406 Saint Martin d'Hères, France

${ }^{j}$ Space Telescope Science Institute, 3800 San Martin Drive, Baltimore, MD 21218, USA

\begin{abstract}
MICADO is the adaptive optics imaging camera for the E-ELT. It has been designed and optimised to be mounted to the LGS-MCAO system MAORY, and will provide diffraction limited imaging over a wide $(\sim 1$ arcmin $)$ field of view. For initial operations, it can also be used with its own simpler AO module that provides on-axis diffraction limited performance using natural guide stars. We discuss the instrument's key capabilities and expected performance, and show how the science drivers have shaped its design. We outline the technical concept, from the opto-mechanical design to operations and data processing. We describe the AO module, summarise the instrument performance, and indicate some possible future developments.
\end{abstract}

Keywords: Adaptive Optics, LGS, MCAO, Imaging, Science Drivers, Astrometry, Photometry, ELT

\section{MICADO OVERVIEW}

MICADO is the Multi-AO Imaging Camera for Deep Observations, designed to work with adaptive optics (AO) on the E-ELT. It has been optimised for the multi-conjugate adaptive optics (MCAO) module MAORY!2 but it is also able to work with other adaptive optics systems, and includes a separate module to provide a single conjugate adaptive optics (SCAO) capability ${ }^{3}$ using natural guide stars during early operations (see Section 44). As this simple AO mode sets low requirements on the telescope and facilities (e.g. no lasers are required), it is an optimum choice for demonstrating the scientific capabilities of the E-ELT at the earliest opportunity. The optical relay and support structure for SCAO provide the same opto-mechanical interface as MAORY, and in principle enable MICADO to be used with other AO systems such as ATLAS! ${ }^{4}$ This phased approach means that MICADO will be able to make use of increasingly sophisticated AO systems as they become available.

MICADO is compact and is supported underneath the AO systems so that it rotates in a gravity invariant orientation. It is able to image, through a large number of selected wide and narrow-band near infrared filters, a large $53^{\prime \prime}$ field of view at the diffraction limit of the E-ELT. MICADO has two arms. The primary arm is a high

Further author information: (Send correspondence to R.D.)

E-mail: davies@mpe.mpg.de 


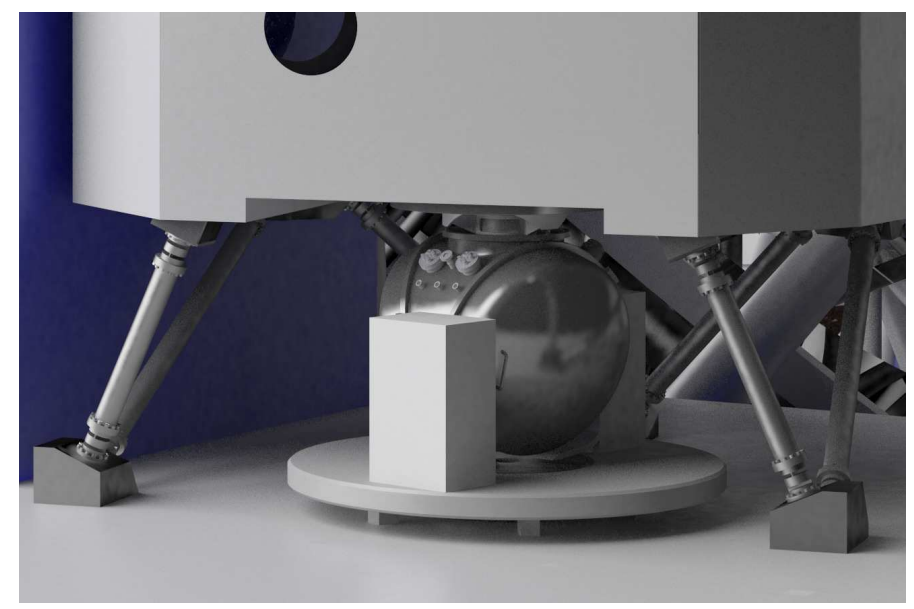

Figure 1. Illustration of MICADO mounted under the MCAO system MAORY on the E-ELT Nasmyth platform.

throughput imaging camera with a single 3 mas pixel scale. This arm is designed with fixed mirrors for superior stability, thus optimizing astrometric precision. In addition, MICADO will have an auxiliary arm to provide an increased degree of flexibility. In the current design, this arm provides (i) a finer 1.5 mas pixel scale over a smaller field, and (ii) a 4 mas pixel scale for a simple, medium resolution, long-slit spectroscopic capability. In principle the auxiliary arm also opens the door to other options, including a 'dual imager' based on a Fabry-Perot etalon to image emission line and continuum wavelengths simultaneously, coronography (perhaps implemented in a comparable way to that in $\mathrm{NACO}^{5}$ ), or a high time resolution detector.

\section{KEY CAPABILITIES AND SCIENCE DRIVERS}

MICADO will excel at several key capabilities that exemplify the unique features of the E-ELT. These are at the root of the science cases, which span key elements of modern astrophysics, and have driven the design of the camera. The science cases are developed in detail elsewhere ${ }^{6}$ and here we focus on how MICADO's characteristics enable it to address them.

\subsection{Sensitivity and Resolution}

MICADO is optimised for imaging at the diffraction limit, and will fully sample the 6-10 mas FWHM in the $J-K$ bands. With a throughput exceeding $60 \%$ its sensitivity at $1-2 \mu \mathrm{m}$ will be comparable to, or surpass, JWST for isolated point sources. MICADO's resolution means that it will be clearly superior to JWST in crowded regions. In addition, its field of view of nearly 1 arcmin yields a significant multiplex advantage compared to other ground-based cameras on ELTs. Together, these characteristics make MICADO a powerful tool for many science cases. Continuum and emission line mapping of high redshift galaxies will enable it to address questions concerning their assembly, and subsequent evolution in terms of mergers, internal secular instabilities, and bulge growth. The resolution of better than $100 \mathrm{pc}$ at $z \sim 2$, equivalent to $1^{\prime \prime}$ imaging of Virgo Cluster galaxies, will resolve the individual star-forming complexes and clusters, which is the key to understanding the processes that drive their evolution. Alternatively, one can probe a galaxy's evolution through colour-magnitude diagrams that trace the fossil record of its star formation. Spatially resolving the stellar populations in this way is a crucial ability, since integrated luminosities are dominated by only the youngest and brightest population. MICADO will extend the sample volume from the Local Group out to the Virgo Cluster and push the analysis of the stellar populations deeper into the centres of these galaxies.

\subsection{Precision Astrometry}

With only fixed mirrors in its primary imaging field, gravity invariant rotation, and HAWAII-4RG detectors (developed to meet the stringent requirements of space astrometry missions), MICADO is an ideal instrument for astrometry. A robust pipeline will bring precision astrometry into the mainstream. An analysis of the statistical 


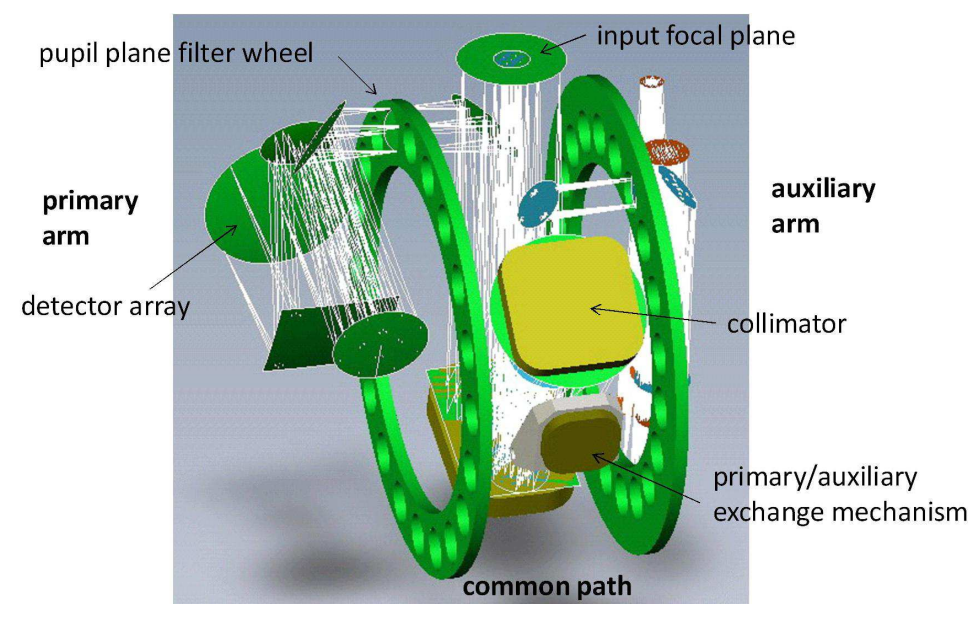

Figure 2. Overview of the MICADO optics. The major components are labelled.

and systematic effects ${ }^{7}$ shows that an accuracy of $40 \mu$ as in a single epoch of observations is achievable; and after only 3-4years it will be possible to measure proper motions of $10 \mu \mathrm{as} \mathrm{yr}^{-1}$, equivalent to $5 \mathrm{kms}^{-1}$ at $100 \mathrm{kpc}$. At this level, many astronomical objects are no longer static but become dynamic, leading to dramatic new insights into the three dimensional structure and evolution of many phenomena. Proper motions of faint stars within light-hours of the Galactic Center will measure the gravitational potential in the relativistic regime very close to the central black hole, and may also reveal the theoretically predicted extended mass distribution from stellar black holes that should dominate the inner region. The internal kinematics and proper motions of Globular Clusters will yield insights on intermediate mass black holes as well as the formation and evolution of the Galaxy. Similar analyses of Dwarf Spheroidals will reveal the amount and distribution of dark matter in these objects, and hence test models of hierarchical structure formation.

\subsection{High Throughput Spectroscopy}

Spectroscopy is an obvious and powerful complement to pure imaging, and is implemented as a simple slit spectrometer with a high throughput that is ideal for obtaining spectra of compact objects. The resolution of $R \sim 3000$ is sufficient to probe between the near infrared $\mathrm{OH}$ lines. This simple addition will enhance many science cases, for example: deriving stellar types and 3D orbits in the Galactic Center; using velocities of stars in nearby galaxies to probe central black hole masses and extended mass distributions; measuring absorption lines in galaxies at $z=2-3$ and emission lines in galaxies at $z=4-6$ to derive their ages, metallicities, and star forming histories; and obtaining spectra of the first supernovae at $z=1-6$.

\section{TECHNICAL DESIGN CONCEPT}

The MICADO design to achieve the capabilites outlined in Section 2 is simple, compact, and robust. As such, it minimizes risks on cost and schedule. In the following, we outline the key features of its design.

\subsection{Optics}

The optics are discussed in more detail elsewhere ${ }^{9}$ and so only a brief outline of the main characteristics is given here. The design was reached after a scientific and technical trade-off. The main requirements from this are that: the pixel scale in the primary arm should be fixed to maximise stability; the scale should be 3 mas to cover a large field of view while being Nyquist sampled in $J$-band; there should be space for a large number of filters; the degree of distortion is less important than its stability (since it must be corrected anyway); the instrument should cope with a strongly curved input wavefront, as well as a flat input wavefront (for a reduced field of view, limited by anisoplanatism, during SCAO operations).

As shown in Fig. 2 the MICADO optics comprises 3 sub-systems: the common path, primary arm, and auxiliary arm. The first component in the common path is a tunable atmospheric dispersion corrector (ADC; 
Table 1. Characteristics of the optical designs for the 2 arms

\begin{tabular}{|c|c|c|}
\hline & Primary Arm & Auxiliary Arm \\
\hline Throughput & $61-70 \%$ for $\mathrm{Y}-\mathrm{K}$ bands & $\begin{array}{l}60-69 \% \text { for imaging in } \mathrm{Y}-\mathrm{K} \text { bands; } \\
18-28 \% \text { for spectroscopy in } \mathrm{Iz}-\mathrm{K} \text { bands }\end{array}$ \\
\hline FoV \& pixel scale & $53^{\prime \prime} \times 53^{\prime \prime}$ at $3 \mathrm{mas} / \mathrm{pixel}$ & $\begin{array}{l}6.4^{\prime \prime} \times 6.4^{\prime \prime} \text { at } 1.5 \mathrm{mas} / \text { pixel (imaging); } \\
17.1^{\prime \prime} \times 17.1^{\prime \prime} \text { at } 4 \mathrm{mas} / \text { pixel (spectroscopy) }\end{array}$ \\
\hline Filters & Single wheel with 20 positions for filters & $\begin{array}{l}\text { Single wheel with } 20 \text { positions for filters } \\
\text { and grisms }\end{array}$ \\
\hline Image quality & $\begin{array}{l}\text { Nominal Strehl ratio at } 0.8 \mu \mathrm{m} \text { is }>83 \% \\
\text { across the whole field }\end{array}$ & $\begin{array}{l}>89 \% \text { for } 1.5 \text { mas imaging; } \\
>75 \% \text { in slit for } 4 \text { mas scale. }\end{array}$ \\
\hline Distortion & $1.2 \%$ across the whole field & $-0.39 \%$ at 4 mas; $0.03 \%$ at 1.5 mas \\
\hline Optical Ghosts & \multicolumn{2}{|c|}{$\begin{array}{l}\text { The reflective optics do no create ghosts; the ADC, entrance window, and filters are } \\
\text { tilted so that ghosts on the detector are minimised }\end{array}$} \\
\hline $\begin{array}{l}\text { Largest Fold } \\
\text { Mirror }\end{array}$ & \multicolumn{2}{|c|}{$260 \times 380 \mathrm{~mm}$ (in common path) } \\
\hline $\begin{array}{l}\text { Largest Working } \\
\text { Mirror }\end{array}$ & $256 \times 276 \mathrm{~mm}$ & $200 \mathrm{~mm}$ diameter (in common path) \\
\hline Tolerances & \multicolumn{2}{|c|}{$0.05 \mathrm{~mm}$ and $0.01^{\circ}$ (i.e. within manufacturable limits) for $70 \%$ strehl at $1 \mu \mathrm{m}$} \\
\hline $\begin{array}{l}\text { Intermediate } \\
\text { Pupil }\end{array}$ & $\begin{array}{l}\text { Shape varies with field position so cold stop } \\
\text { is undersized at } 99.1 \mathrm{~mm} \text {. Maximum vi- } \\
\text { gnetting is } 1.0 \% \text { in corner of field }\end{array}$ & $\begin{array}{l}\text { Cold stop undersized at } 85.6 \mathrm{~mm} \text {. } \\
\text { Maximum vignetting is } 0.4 \%\end{array}$ \\
\hline Focal Plane & $\begin{array}{l}264 \times 268 \mathrm{~mm} \text {; tilted by } 4.1^{\circ} \text { and convex } \\
\text { with a } 1500 \mathrm{~mm} \text { curvature radius (due to } \\
\text { input wavefront curavature) }\end{array}$ & $\begin{array}{l}61.4 \mathrm{~mm} \text { across; } \\
\text { unaffected by input curvature }\end{array}$ \\
\hline $\begin{array}{l}\text { SCAO image } \\
\text { quality }\end{array}$ & $\begin{array}{l}\text { Strehl ratio is }>84 \% \text { at } 0.8 \mu \mathrm{m} \text { over full } 27^{\prime \prime} \\
\text { SCAO field }\end{array}$ & $>78 \%$ \\
\hline
\end{tabular}

not shown in the figure). It consists of 2 pairs of $\mathrm{ZnSe} / \mathrm{ZnS}$ prisms that can be rotated to provide a dispersion correction that is optimised to the observational band. It is located as far in front of the input focal plane as possible (i.e. $500 \mathrm{~mm}$ ). This is to minimize its impact on the optical quality by enabling thinner prisms with smaller wedge angles to be used. In its current location (warm and right against the interface to MAORY), the performance is just acceptable. However, this location is not optimal and during Phase B other options, such as locating it at an appropriate pupil plane within the AO system, will be addressed.

Both arms use an off-axis parabola for collimation. However, the sizes and locations of the parabolae are different, and so the primary arm has a fixed mirror, while the auxiliary arm requires an alternative mirror to be rotated into position. In both cases, to keep the optical system compact, the light is reflected in both directions from a large fold mirror. Separate fixed fold mirrors then direct the light out of the common path to opposite sides of the instrument. The collimator creates a pupil image just after this fold mirror, where a large filter wheel is located. The maximum circular diameter of the pupil is $100.5 \mathrm{~mm}$; however, in order to block unwanted thermal background (the pupil has a shape that depends slightly on field position), the coldstop is undersized at $99 \mathrm{~mm}$ diameter. The primary arm has only 3 additional working mirrors (based on a TMA), although extra fold mirrors are required to keep the volume occupied small. The auxiliary arm is similar, but includes mechanisms for changing the pixel scale. Table 1 summarizes the key characteristics of the two arms.

The baseline detector for MICADO is the HAWAII-4RG. These have a number of important advantages: (i) they are large format, so that relatively few detectors need to be characterised and mosaiced, (ii) they have been designed for the stringent requirements of space astrometry missions and so are ideal for MICADO's astrometry applications, (iii) the readout speed can be adjusted (even on multiple sub-regions), greatly reducing the impact of saturation due to bright targets. The cross-talk between pixels is relatively low and electronic ghosts can largely be suppressed in the same way as is done for XShooter. There will be relatively small gaps (each a few $\mathrm{mm}$ compared to the $25 \mathrm{~cm}$ width of the focal plane) between individual detectors since they are not directly buttable. This can be considered advantageous since it provides a quasi-coronagraph, allowing one to position a bright star out of the field of view even in dithered exposures. To optimise stability of the focal plane array, all 

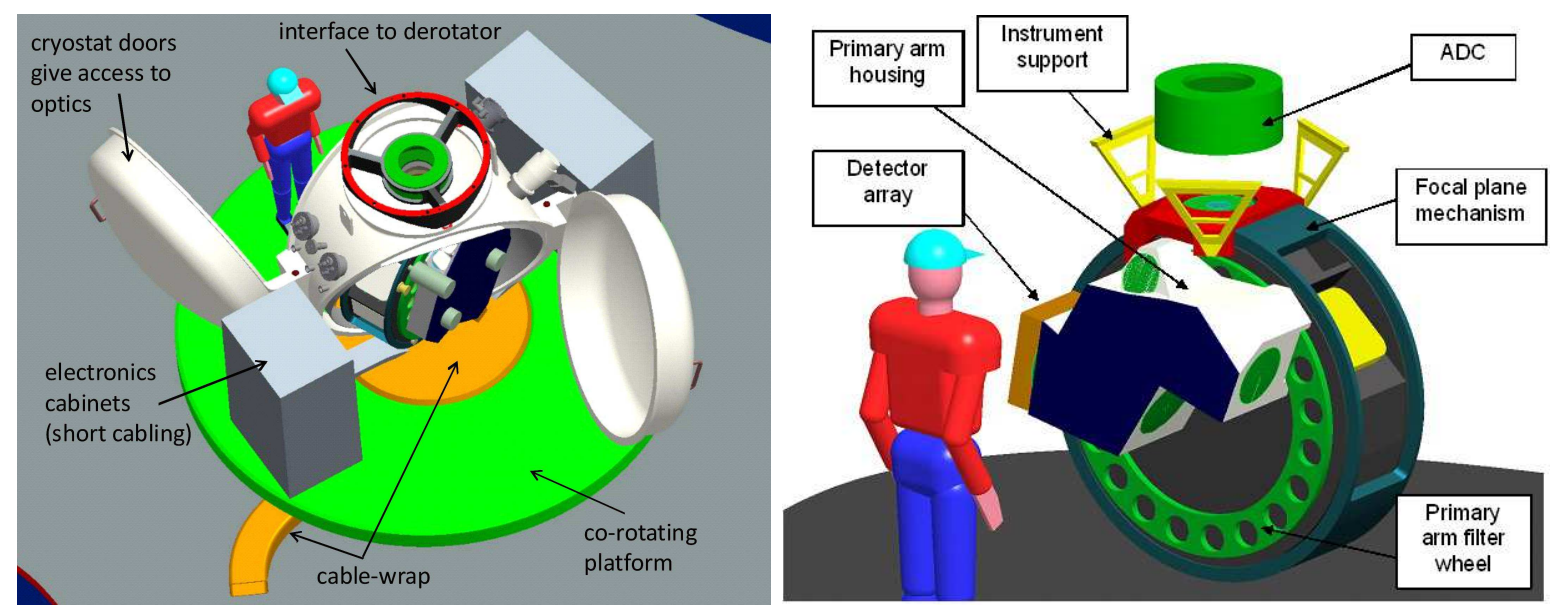

Figure 3. Left: The main components of MICADO, with the cryostat access doors open. Right: overview of the MICADO mechanical structure, with the main structures labelled.

detectors will be mounted on a single baseplate. A direct result of the strongly curved input focal plane from the MCAO system (which cannot be corrected in a satisfactory way) is that the MICADO focal plane is tilted by $4.1^{\circ}$ and curved, with a $1500 \mathrm{~mm}$ radius of curvature. Because the $60 \mathrm{~mm}$ wide detectors are flat, there will be a small defocus across each detector due to the $\pm 0.32 \mathrm{~mm}$ focal plane mismatch. This has only a minor impact on the spot diagrams, and the Strehl ratio at $0.8 \mu \mathrm{m}$ remains above $88 \%$ across the whole field (i.e. significantly larger than that used for tolerancing).

\subsection{Mechanics}

The mechanical design, and folding of the optical path, has largely been driven by the limited space under MAORY. To keep torques small and to maintain optical alignment during cool-down, the centre of gravity is close to the optical axis, which itself is close to the centre of shrinkage. In order to minimize cable lengths and to limit the mass mounted on the derotator, the electronics racks stand on a co-rotating platform supported on the Nasmyth floor. This platform also houses the cable-wrap for external supplies. Service and maintenance are also key aspects, leading to a design in which the core instrument and optics structure are rotated by $25^{\circ}$ with respect to the cryostat. This provides better access through the cryostat doors to the detector arrays, the arm selection and focal plane mechanisms, the filter wheels, and the core optics.

MICADO is housed in a stainless steel cryostat (Fig. 3, left), which has a fixed tapered part with sufficient space for all the through-ports and pumps. On either side are 2 large doors which provide access to all key components while MICADO is mounted to the AO system. The 3 electronics cabinets ( 2 of which are backto-back) are positioned on the co-rotating platform in such a way that they do not interfere with the doors. The entrance window of the cryostat is located $300 \mathrm{~mm}$ above the focal plane and $200 \mathrm{~mm}$ below the mounting interface. The warm ADC is currently located in this volume for the reasons outlined in Section 3.1. To minimise flexure (which is critical for astrometry), the instrument has been designed for gravity invariant rotation. Because of this, and since the cool-down times are limited by thermal contact of the filters, the cryostat has not been lightweighted. The total mass of the cryostat and instrument supported by the derotator is $3000 \mathrm{~kg}$. An additional $2800 \mathrm{~kg}$ are supported on the Nasmyth floor, and $500 \mathrm{~kg}$ in a calibration unit located in the AO system.

Supported inside the cryostat behind the radiation shield is the cold optics instrument (Fig. 3, right). It comprises 3 main structures: the primary arm, auxiliary arm, and core sub-assembly. The general design approach for each of these housings is to assemble them from plate material to keep part complexity and accuracy low, and ensure a rigid boxed structure. Stray light can be reduced by proper shielding and baffling, and also by using a wave-like finish to the surface of walls, and applying a low-reflectivity black coating. The filter wheels for each of the arms are mounted on the side panels of the core structure, and supported at their perimeter. The primary and auxiliary arm housings are mounted to the core with a three-point interface in such a way that they 


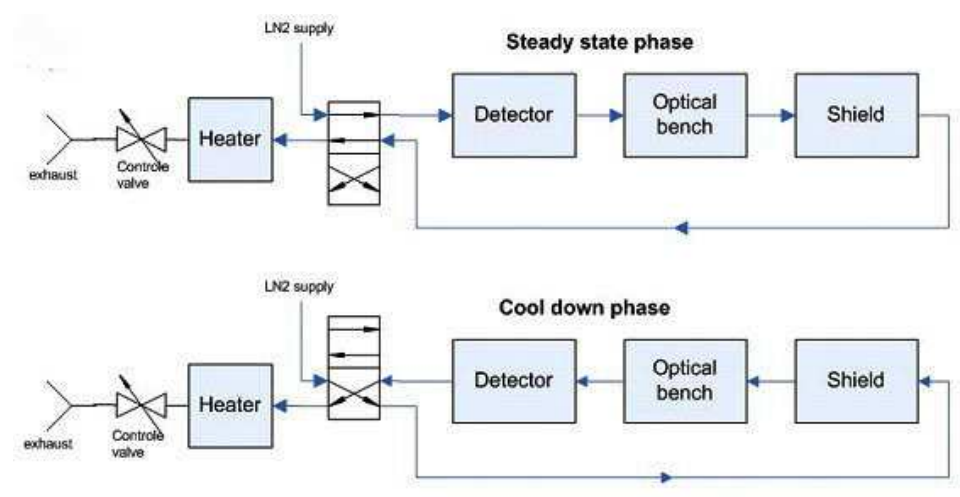

Figure 4. Overview of the MICADO reversible cryogenic scheme

connect to the round side panels with direct support underneath from the core wall panels. The instrument core is supported by the cryostat via $3 \mathrm{~V}$-rods and a transfer structure. The rods are co-axially aligned to maintain alignment during cool-down, and are attached to the transfer structure. This has been designed to accommodate the rotating focal plane mechanism, and acts as a bridge to the stiff support structure of the core subassembly. MICADO has relatively few cold mechanisms. In order to achieve high repeatability, these are all rotational and use spring loaded bearings and V-grooves to locate and lock each position:

Focal plane selection: the input focal plane is large, and 6 positions are required (field stop for each arm, 2 long slits, a closed position, and a point source mask for initial check of internal focus). It is a large structure that is driven around the outside of the core sub-assembly.

Primary/Auxiliary Arm selection: the core contains only one mechanism, which rotates in an alternative parabolic collimator mirror if the auxiliary arm is selected. Because this is recognised as being delicate, accessibility is an important design driver, and is facilitated by a large opening in the focal plane wheel.

Filter wheels: The pupil sizes are $100 \mathrm{~mm}$ and $86 \mathrm{~mm}$ diameter for the primary and auxiliary arms. In order to provide space for 20 filter slots, these wheels are large, and hence supported and driven at their perimeter. Since the filters will dictate the cool-down time, care has been given to maximising the thermal contact.

Scale changing mechanism: the auxiliary arm has two mechanisms to move mirrors and enable a pixel scale change. The design is similar to the primary/auxiliary selection mechanism.

\subsection{Cryogenics}

In order to avoid the use of cryo-coolers, the vibrations from which would have a strong adverse effect on the AO performance, MICADO will be cooled by continuous flow liquid nitrogen (LN2) during cool-down/warmup cycles as well as steady state phases. For cooldown, an estimated $1000 \mathrm{~L}$ will be needed; and to maintain steady-state the required flow rate, including some contingency, is expected to be $72 \mathrm{~L} /$ day.

Continuous flow is preferred over a LN2 bath since it gives more freedom in the location of the detectors, it keeps the cryostat smaller and its mass lower, and it is possible to combine the precooling and steady state systems. Cooling pads are located at strategic points in the cryostat, and connected so that during a cool-down cycle the heat shield is cooled first, followed by the optical bench and finally the detectors. During steady state the sequence is reversed so that the detectors have the lowest possible temperature. This series concept is shown in Fig. [4 which demonstrates how the same circuit can be used during both phases.

\subsection{Electronics}

The electronics cabinets are physically located very close to the MICADO cryostat, on a platform that co-corates with it. This means that the cables can be kept short. At the current time, no ELT electronics standards have been defined. The preferences for MICADO include the architecture being based on SIMATIC Programmable Logic Controller (PLC), Realtime Ethernet or other Realtime architectures. A fail-safe version of the PLC SMATIC 57 is also an option for cryogenic housekeeping (cryogenics control). And Realtime LabView and PXI controller from National Instruments could also be used in the implementation of control electronics. 


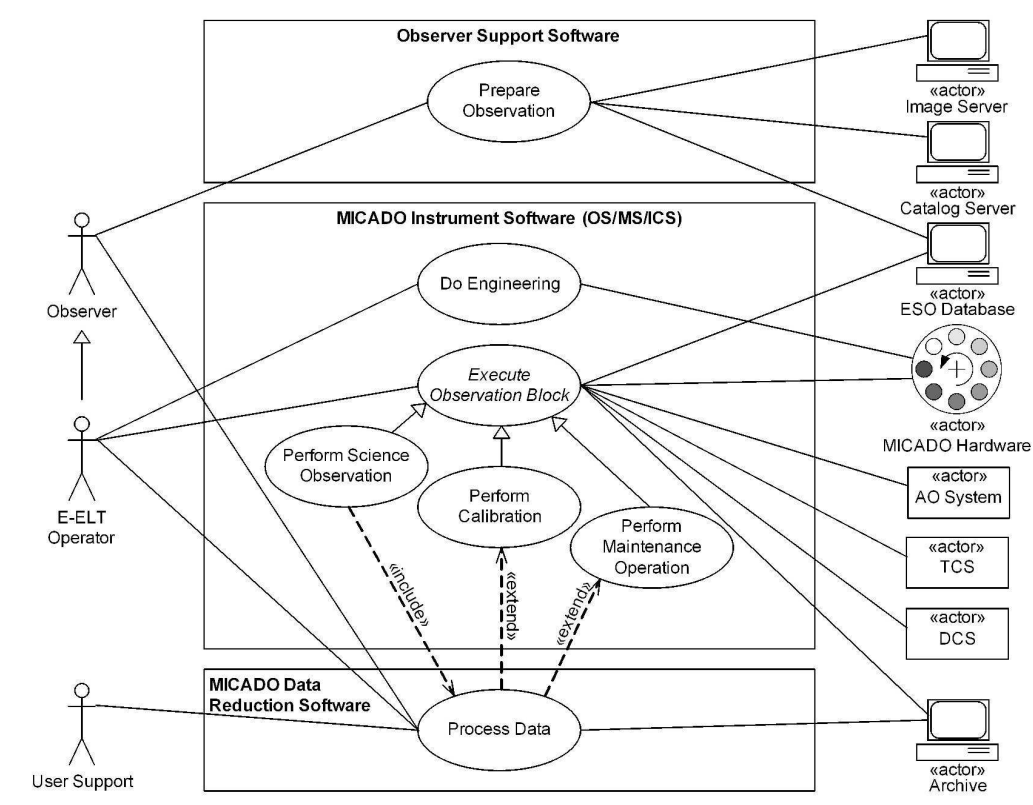

Figure 5. Overview of top-level use cases and actors for the MICADO instrument control software

\subsection{Instrument Control}

The user requirements for the instrument software have been developed for observation preparation, science operations (including on-sky calibration), and maintenance operations. The main functionality has been analysed via specific use cases related to the various observing scenarios and modes. An overview of the complete scheme is given in Fig. [5] Specific use cases include:

Science Observations: imaging, spectroscopy, on-sky calibrations (e.g. standard stars, twilight flats)

Calibrations: dark frames, internal flatfield, linearity, wavelength \& distortion calibrations, ghost assessment

Maintenance Operations: telescope focus.

It is likely that, apart from the actual instrument control software itself, MICADO will share a common software layer with other E-ELT subsystems. However, at the current time, no E-ELT software standards have been defined. Given the timeline of the project and the need for a stable development infrastructure, open source solutions are preferred over proprietary commercial ones in this context. For the same reason, Linux is favoured as the operating system.

\subsection{Data Processing}

The user requirements for the data reduction software have been developed from observational scenarios for imaging and spectroscopy. These are standard techniques, and lead to no surprises. The performance required for photometry and astrometry have led to additional requirements. These include the use of a special internal calibration mask to measure instrument distortions, and additional steps in the data processing for the related science projects. The Astro-WISE system is well suited for reduction of MICADO data. Astro-WISE is an integrated system where users cannot only perform data reduction but also data archiving, post-reduction analysis and publishing of the raw, intermediate and final data products. A salient feature for the data reduction relevant for MICADO is that it performs 'global' astrometry and photometry. Astrometric and photometric corrections and calibrations by combining the information from overlapping observations improving on calibrations based on individual pointings. Data reduction can take place in fully automated fashion or in a more manual fine-tuning manner. The data rates estimated for MICADO are up to about 6 Terabytes per night (if all individual exposures are kept and processed for optimal astrometric accuracy); although significantly less if either short exposures can be directly co-added in the detector control system (DCS), or longer exposures are required (e.g. when using narrow band filters). 


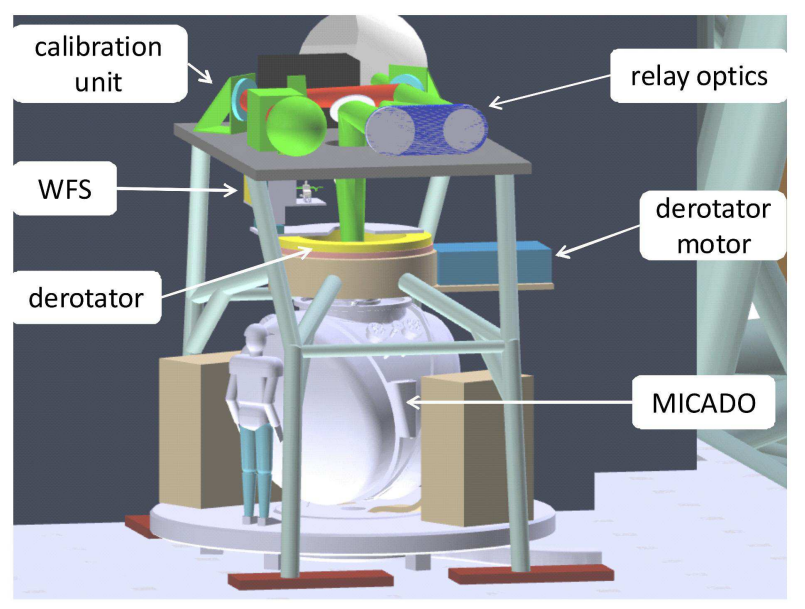

Figure 6. MICADO SCAO module 'SAMI'.

\section{ADAPTIVE OPTICS}

The design of MICADO has been optimised for the multi-conjugate adaptive optics module MAORY uses multiple lasers and natural guide stars to provide diffraction limited performance over a wide field with high sky coverage. However, the Phase A study included a simpler AO system that can be used during initial operations, in order to mitigate the risk associated with such a complex AO system, and to enable MICADO to produce diffraction limited images at the earliest opportunity. This AO system will be single-conjugate and use a single natural guide star as the wavefront reference: there will be sufficient science targets near suitable guide stars for 2-3 years of operation. Including SCAO in the MICADO design is necessary because, at the current time, the E-ELT baseline does not include a wavefront sensing capability for scientific instruments (although any WFS can make use of the E-ELT's deformable and tip-tilt mirrors). And because MICADO cannot interface to the Nasmyth port, a major part of the SCAO system is an optical relay and support structure that provides the same mechanical and operational interface as to MAORY. Since it can in principle be re-used with other AO systems such as ATLAS, $\stackrel{4}{4}$ this means that MICADO will be able to make use of increasingly sophisticated AO systems as they become available.

The top level requirements for the SCAO module are relatively simple: the optical, mechanical, and communication interfaces should be the same as those to/from MAORY; the WFS bandpass should be $0.45-0.8 \mu \mathrm{m}$, to maximise sensitivity without compromising the scientific wavelength range; the WFS should be able to guide on stars anywhere within a $45^{\prime \prime}$ diameter patrol field; and the transmitted scientific field of view need only be $27^{\prime \prime} \times 27^{\prime \prime}$ (commensurate with the isoplanatic patch size). This last requirement means that even though there is no field curvature from the telescope (in contrast to the strongly curved field from MAORY for which MICADO is designed), the image quality over the SCAO field is unaffected. It also means that initially, only the central detectors need be mounted; the remainder can be integrated later. SAMI, the SCAO module for MICADO shown in Fig. 6] is described in detail elsewhere $\stackrel{3}{3}^{1}$ It comprises 4 sub-systems:

an optical relay made of a 3-mirror Offner relay, a folding mirror directing the light downward to MICADO and the WFS, and a dichroic plate splitting the light between MICADO and the WFS.

a field derotator to compensate for the telescope movements while tracking, for both MICADO and the WFS. a support structure for the optical bench of the relay optics, the WFS, the derotator and MICADO.

the WFS, including all opto-mechanics after the dichroic. It comprises a pupil steering mirror, and, mounted on XY stages, a field stop, a K-mirror for pupil derotation, a lens triplet and the WFS camera itself. The initial study suggests that an ADC in the WFS should not be necessary.

The performance of the MICADO SCAO module has been estimated using analytical formulae (e.g. for the anisoplanatism error), information from ESO (e.g. for the fitting error) and two home-made simulation tools. 
It takes into account the control laws that will be implemented: a classical integrator, with a modal control, together with a Kalman filter for windshake compensation; and also that smart algorithms, such as weighting or pixel selection, will be used for the centre of gravity computation. The results of these computations are summarized in Tables 2 and 3. Multiplying values from both tables together will yield an estimation of the strehl ratio expected for a guide star of a given magnitude at a given distance off-axis.

Table 2. SCAO performance as a function of guide star magnitude

\begin{tabular}{|l|c|c|c|c|c|}
\hline On-axis reference source V-band magnitude & 12 & 13 & 14 & 15 & 16 \\
\hline Total wavefront error $(\mathrm{nm} \mathrm{rms})$ & 183 & 205 & 245 & 328 & 514 \\
\hline Strehl at $2.2 \mu \mathrm{m}$ & $76 \%$ & $71 \%$ & $61 \%$ & $41 \%$ & $12 \%$ \\
\hline
\end{tabular}

Table 3. Anisoplanatic effect on strehl ratio for SCAO

\begin{tabular}{|l|c|c|c|c|c|c|}
\hline Distance from reference source $(\operatorname{arcsec})$ & 5 & 15 & 25 & 35 & 45 & 55 \\
\hline Anisoplanatism error for L0=25m (nm rms) & 101 & 253 & 354 & 433 & 518 & 554 \\
\hline Corresponding Strehl ratio scaling at $2.2 \mu \mathrm{m}$ & $92 \%$ & $59 \%$ & $36 \%$ & $22 \%$ & $11 \%$ & $8 \%$ \\
\hline
\end{tabular}

\section{OPERATION AND CALIBRATION}

The basic operational scenario for MICADO is very similar to other imaging cameras and spectrometers. For imaging, the sky background will be derived either by combining dithered exposures or, when necessary, by offsetting to sky. For spectroscopy, the source will be nodded back and forth along the slit. Typical exposure times will be a few seconds (broad band filters) up to tens of seconds (narrow band filters). For the shortest exposure times, several exposures will be made at the same pointing before dithering. The main issue is the size of the dithers, which must be optimised for science while minimizing the AO and telescope overheads. Table 4 summarises the definition of dithers and offsets for MICADO.

Table 4. Definition of dithers and offsets to reduce AO and telescope overheads

\begin{tabular}{|l|l|}
\hline Small dither & $\begin{array}{l}\text { Offset of up to } \pm 0.3^{\prime \prime}\left(\text { goal } \pm 0.5^{\prime \prime}\right) \text { from initial pointing in each of X- and Y- directions, with } \\
\text { an accuracy of }<2 \text { mas. AO loops remain closed during operation. Cadence: } 10-30 \text { sec. }\end{array}$ \\
\hline Large dither & $\begin{array}{l}\text { Offset of up to } \pm 10^{\prime \prime} \text { from the initial pointing. AO loops open during the offset, but reclose } \\
\text { at the new position. The telescope is involved. Cadence: a few minutes. }\end{array}$ \\
\hline Sky Offset & $\begin{array}{l}\text { Offset of up to } 15^{\prime} \text { (when background cannot be recovered by dithering). AO loops do not } \\
\text { need to close in the new position. Cadence: } 10-30 \text { minutes (depending on overhead). }\end{array}$ \\
\hline Sky Return & Offset back, after a 'Sky Offset'. AO loops should reclose. \\
\hline
\end{tabular}

Most of the calibrations can be performed internally during the day while the dome lights are on: flatfields, wavelength calibration, darks. Additional twilight flats will be required in order to correct illumination gradients in the internal flats. The only non-standard calibration required is that to correct instrument distortions in the AO system and MICADO. This will also be possible during the day with the dome lights on, and will be achieved by inserting a special calibration mask into the focal plane in front of the AO system. The only standard nighttime calibration is to observe standard stars for flux calibration.

\subsection{Astrometric Calibration}

In the Galactic Centre, it is possible to achieve a relative astrometric precision of $200-300 \mu$ as $\sqrt{10}$ in the $H$-band on an 8-m class telescope. This corresponds to about $0.5 \%$ of the FWHM of the PSF. If this performance is projected forward to the E-ELT, one can hope to reach a precision of about $40 \mu \mathrm{as}$. In Table 5 we summarise the conclusions from a study ${ }^{8}$ of the ten error sources that need to be controlled in order to achieve this. Measuring these effects clearly requires careful calibration, and the scheme outlined in Fig. 7 shows how this can be done. An internal calibration mask is used to measure discontinuities between the detectors and instrumental distortions in order to map the detector plane onto the sky. This provides a set of relative (or artifical) coordinates to which subsequent exposures can be matched by applying a low order transformation based on point sources in the field. Exposures within a single epoch can then be combined. Deep integrations obtained in this way at different epochs are mapped to each other via another low order transformation, using either faint compact galaxies (which have 


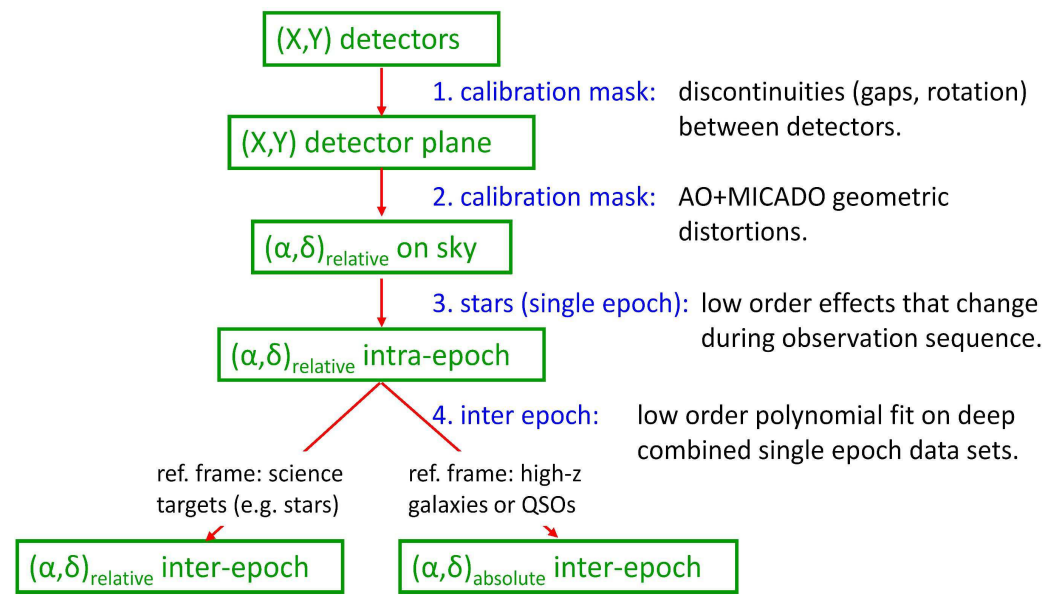

Figure 7. Astrometric calibration for MICADO is achieved in several stages.

negligible proper motions) or by using the ensemble of stars. In the latter case, their relative proper motions (a high order effect) are preserved during a low order transformation.

Table 5. Sources of astrometric uncertainty

\begin{tabular}{|l|l|}
\hline Absolute plate scale & $\begin{array}{l}\text { pre-imaging (seeing limited) can give better than } 10^{-4} \text { accuracy (i.e. 5 mas over } \\
50^{\prime \prime} \text { field). }\end{array}$ \\
\hline Sampling \& pixel scale & $\begin{array}{l}\text { no measurable errors if pixel scale does not exceed } 3 \text { mas pix }{ }^{-1} \text { (note that a finer } \\
\text { scale is beneficial for highly crowded fields). }\end{array}$ \\
\hline $\begin{array}{l}\text { Instrumental } \\
\text { distortions }\end{array}$ & $\begin{array}{l}\text { are measured to } 0.01 \text { pixel in many current instruments. For MICADO, calibration } \\
\text { with an internal mask will reduce this error to } \sim 30 \mu \text { as. }\end{array}$ \\
\hline Telescope instabilities & $\begin{array}{l}\text { plate scale, rotation, etc., are low order effects that can be absorbed into a coor- } \\
\text { dinate transformation. }\end{array}$ \\
\hline $\begin{array}{l}\text { Achromatic differential } \\
\text { refraction }\end{array}$ & this large $\sim 10$ mas effect is linear and so is removed by a coordinate transformation. \\
\hline $\begin{array}{l}\text { Chromatic differential } \\
\text { refraction }\end{array}$ & $\begin{array}{l}\text { produces } ~ 1 \text { mas scale effects that depend on the source colour. A tunable ADC } \\
\text { can reduce it to }<20 \mu \text { as in most cases. }\end{array}$ \\
\hline $\begin{array}{l}\text { AO instrumental \& } \\
\text { atmospheric effects }\end{array}$ & $\begin{array}{l}\text { shift the relative positions of the NGS used by the AO system. MAORY uses 3 } \\
\text { NGS and so this is expected to be a low-order effect. Since they are slow effects, } \\
\text { they can also be removed by tracking the barycentre of each NGS. }\end{array}$ \\
\hline Differential tilt jitter & $\begin{array}{l}\text { introduces errors of } \sim 100 \mu \text { as into diffraction limited E-ELT observations. It scales } \\
\text { as } t^{-1 / 2} \text { and can be integrated down to } \sim 10 \mu \text { as within about 30 min. }\end{array}$ \\
\hline PSF variations & $\begin{array}{l}\text { even with MCAO the PSF changes across the field of view. Measurements on } \\
\text { simulated PSFs indicate this should intrdouce errors of }<10 \mu \text { as. }\end{array}$ \\
\hline $\begin{array}{l}\text { Galaxies as astromet- } \\
\text { ric references }\end{array}$ & $\begin{array}{l}\text { galaxies are spatially resolved, but making use of their detailed internal structure } \\
\text { enables one to reach } \sim 20 \mu \text { as accuracy with deep integrations. }\end{array}$ \\
\hline
\end{tabular}

\subsection{PSF Calibration and Photometry}

The precision with which photometry can be performed is determined by the accuracy to which the PSF is known. This issue is being addressed in 2 complementary ways. The MAORY consortium are developing a simple model for the PSF which enables its shape to be determined with only a few parameters. In principle, one can map the variation of each parameter across the field of view. With relatively few empirical measurements, this might yield a quantitative estimate of the PSF at any point. Such a tool would be extremely important for many science cases. In particular, for studying black hole and host galaxy growth across cosmic time, an accurate estimate of the PSF is needed in order to separate the QSO and host galaxy emission. In crowded 


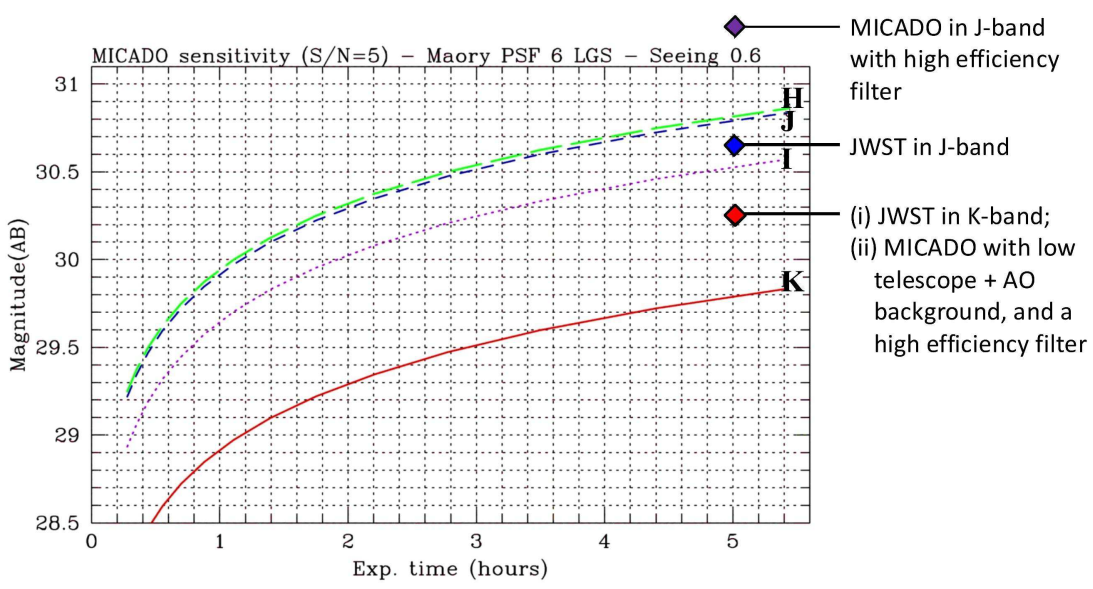

Figure 8. MICADO sensitivity (as a function of integration time) for broad-band imaging through standard filters. A few reference points for $5 \mathrm{hr}$ integrations are shown for comparison.

stellar fields, simulations indicate that it is possible to derive the PSF from the data itself and perform accurate photometry using currently available tools. This works over a small field where PSF variations are negligible. For MCAO, simulations indicate that although spatial variations in the PSF are small, they will have some impact on photometric accuracy. Therefore, to cover a larger field, one would either need to stitch together multiple sub-fields that are analysed separately; or develop the photometry tools to cope with a spatially variable PSF.

\section{PERFORMANCE}

The broadband imaging performance for the MICADO primary field is show in Fig. 8 . This has been calculated for isolated point sources using PSFs provided by the MAORY consortium and for standard broadband filters similar to those in HAWK-I. It shows that the $5 \sigma$ sensitivity will be better than a few nano-Jy (30 mag AB) for the $I-H$ in only $1-2$ hours. The $K$-band performance depends strongly on the thermal background and hence the ambient temperature, but is likely to be about 1 mag less. Advanced filters (see Section 7) will have a very significant impact on MICADO sensitivity. A prototype $J$-band filter pair increases the sensitivity in a given integration time by $0.3 \mathrm{mag}$. More advanced design optimisation techniques could lead to a 0.5 mag sensitivity gain in this band, and comparable gains may be expected for the $I$-band and $H$-band.

Table 6. Sensitivity (AB mag) for isolated point sources to $5 \sigma$ in 5 hours

\begin{tabular}{|l|c|c|c|}
\hline & $J_{A B}$ & $H_{A B}$ & $K_{A B}$ \\
\hline Imaging & 30.8 & 30.8 & 29.8 \\
Imaging with advanced filters & 31.3 & 31.3 & 30.2 \\
Spectroscopy between the OH lines & 27.2 & 27.2 & 25.7 \\
\hline
\end{tabular}

The spectroscopic performance has been calculated for isolated point sources that are nodded back and forth along a slit that is $8^{\prime \prime}$ long and 12 mas wide. Because of the unusually extreme core+halo shape of the adaptive optics PSF, this width maximises the signal-to-noise reached for point sources in the $J$ and $H$-bands. In the $K$-band, additional diffraction losses at the slit reduce the throughput slightly. The sensitivity calculation takes account of all effects (including the Strehl ratios predicted by MAORY, the limited coupling efficiency due to the PSF shape, diffraction losses at the slit, and the thermal background). The resulting $5 \sigma$ sensitivities are $J_{A B}=H_{A B}=27.2 \mathrm{mag}$ between the $\mathrm{OH}$ lines in a 5 hour integration; and similarly $K_{A B}=25.7 \mathrm{mag}$ (which is, as before, less primarily due to the thermal background).

\section{TECHNOLOGICAL DEVELOPMENTS AND RISKS}

MICADO is a simple camera and has been designed to have few risks. Indeed, the preliminary risk register contains no technical or programmatic risks above a low level. Those that do exist at this level are common risks 
associated with all (cryogenic) instruments, and not specific to MICADO. There are several future developments that could be beneficial. Although none of these is required for the successful functioning of MICADO, they would each increase the competitiveness of MICADO with respect to other facilities. The developments include:

Advanced filters: Substantial gains in sensitivity of ground-based near-infrared instruments can be attained by sky line suppression or avoidance. We have begun a research project with Laser Zentrum Hannover to develop high throughput broad band filters and $\mathrm{OH}$ suppressing interference filters. The initial work, nearing completion, is to make a prototype for the $J$-band comprising low-pass and high-pass filters coating opposite sides of a substrate. Together these make a broad-band filter with $>95 \%$ throughput (filters with 96-99\% throughput, but suppression over a shorter baseline, were already maunfactured several years agd $\left.{ }^{11}\right)$. The $\mathrm{OH}$ suppression is achieved by transmitting several narrow bandpasses within this range where the background is sufficiently low. Future development will focus on extension to other bands, optimisation of the filter profile, process qualification, and coating homogeneity, stress, and characterisation.

Dual Imager: Fabry-Perots are complementary to integral field spectroscopy, but provide higher quality images (greater fidelity, and higher resolution over a larger field) of individual emission lines. The key to success would be to enable simultaneous imaging of emission line and continuum wavelengths. This would avoid problems with variable seeing or AO performance when subtracting the continuum to obtain the line emissiom map. Some development is required to achieve a good optical design.

High Time Resolution Astronomy 12 Scientific applications include the stochastic behaviour of neutron stars and white dwarf accretion disks, and pulsar magnetospheres; and time resolved observations of gamma-ray and $\mathrm{X}$-ray transients and anomalous repeaters. Detector technology is available now in the range $0.8-1.2 \mu \mathrm{m}$ using avalanche photodiodes (APDs) and pnCCDs, $\frac{13}{13}$ and there is every expectation that it will extend towards $2 \mu \mathrm{m}$ over the next few years. A high time resolution instrument is essentially an imaging device with a fast detector, and so there would be very little additional opto-mechanical development required to include such a capability in MICADO's auxiliary arm.

\section{REFERENCES}

1. Diolaiti E., et al., in Adaptative Optics for Extremely Large Telescopes, eds. Y. Clénet, T. Fusco, G. Rousset, (EDP Sciences) id.02007 (2010)

2. Foppiani I., et al., in Adaptative Optics for Extremely Large Telescopes, eds. Y. Clénet, T. Fusco, G. Rousset, (EDP Sciences) id.02013 (2010)

3. Clénet Y., et al., in Adaptive Optics Systems II eds B. Ellerbroek, M. Hart, N. Hubin, P. Wizinowich, Proc SPIE, 7736 (2010)

4. Fusco T., et al., in Adaptative Optics for Extremely Large Telescopes, eds. Y. Clénet, T. Fusco, G. Rousset, (EDP Sciences) id.02002 (2010)

5. Boccaletti A., et al., PASP, 116, 1061 (2004)

6. Renzini A., et al., in MICADO Phase A Scientific Analysis Report, E-TRE-MCD-561-0007, issue 2.0 (2009)

7. Davies R., et al., in Adaptative Optics for Extremely Large Telescopes, eds. Y. Clénet, T. Fusco, G. Rousset, (EDP Sciences) id.01002 (2010)

8. Trippe S., Davies R., Eisenhauer F., Förster Schreiber N.M., Fritz T., Genzel R., MNRAS, 402, 1126 (2010)

9. Magrin D., et al., in Ground-based and Airborne Instrumentation for Astronomy III, eds I. McLean, S. Ramsay, H. Takami, Proc SPIE, 7735 (2010)

10. Fritz T., et al., MNRAS, 401, 1177 (2010)

11. Günster S., Ristau D., Greiner J., Tafelmaier C., in Advances in Optical Thin Films III, eds N. Kaiser, M. Lequime, A. Macleod, Proc SPIE, 7101, id.71011V (2008)

12. Shearer A., et al., in High Time Resolution Astrophysics: The Universe at Sub-Second Timescales, eds D. Phelan, O. Ryan, A. Shearer, AIP Conf. Proc., 984, pp.225-232 (2008)

13. Hartmann R., et al., in Adaptive Optics Systems, eds N. Hubin, C. Max, P. Wizinowich, Proc. SPIE, 7015, id.70155C (2008) 